Mainz preprint KOMA-96-32

July 1996

\title{
Three-dimensional 3-state Potts model revisited with new techniques
}

\author{
Wolfhard Janke ${ }^{1}$ and Ramon Villanova ${ }^{2}$ \\ ${ }^{1}$ Institut für Physik, Johannes Gutenberg-Universität Mainz, \\ Staudinger Weg 7, 55099 Mainz, Germany \\ 2 Matemàtiques Aplicades, Universitat Pompeu Fabra, \\ La Rambla 32, 08002 Barcelona, Spain
}

\begin{abstract}
We report a fairly detailed finite-size scaling analysis of the firstorder phase transition in the three-dimensional 3-state Potts model on cubic lattices with emphasis on recently introduced quantities whose infinite-volume extrapolations are governed only by exponentially small terms. In these quantities no asymptotic power series in the inverse volume are involved which complicate the finite-size scaling behaviour of standard observables related to the specific-heat maxima or Binderparameter minima. Introduced initially for strong first-order phase transitions in $q$-state Potts models with "large enough" $q$, the new techniques prove to be surprisingly accurate for a $q$ value as small as 3. On the basis of the high-precision Monte Carlo data of Alves et al. [Phys. Rev. B43 (1991) 5846], this leads to a refined estimate of $\beta_{t}=0.550565(10)$ for the infinite-volume transition point.
\end{abstract}

PACS numbers: $05.50 .+\mathrm{q}, 75.10 . \mathrm{Hk}, 64.60 . \mathrm{Cn}$

e-mail: janke@miro.physik.uni-mainz.de

WWW: http://www.cond-mat.physik.uni-mainz.de/ janke 


\section{Introduction}

The three-dimensional (3D) 3-state Potts ferromagnet serves as an important model in both condensed matter as well as high-energy physics [1]. Experimental realizations are structural phase transitions in some crystals, and theoretically this model has attracted much interest as a simple effective model of finite-temperature pure-gauge QCD. Consequently it has been studied in the past few years by many authors using quite a variety of different techniques [2-23]. By analyzing Monte Carlo (MC) simulations with the help of standard finite-size scaling (FSS) methods [24-27] the characteristic parameters of the phase transition (transition temperature, latent heat, etc. ) have been estimated with varying accuracy. As a result there is by now general consensus that this model undergoes on a simple cubic lattice a weak firstorder phase transition from a three-fold degenerate ordered low-temperature phase to a disordered phase at high temperatures.

Most high-precision MC results are based on the FSS of the specificheat maxima, the Binder-parameter minima, or the partition function zeros. For some of these observables the pseudo-transition points, $\beta_{t}(L)$, exhibit a non-monotonic FSS behaviour with a peculiar dip around linear lattice sizes $L=30$ for, e.g., the specific-heat data. This clearly indicates that for the standard observables the asymptotic FSS region is not reached until $L>30$. For large systems with periodic boundary conditions, as a consequence of phase coexistence at a first-order phase transition, the FSS behaviour of these observables is governed by asymptotic series expansions in inverse powers of the volume $V=L^{D}$ [24-27]. In addition there are also further corrections that decrease exponentially with the system size $[28,29]$. They originate from finite-size effects $\propto \exp \left(-L / L_{0}\right)$ in the pure phases, where $L_{0}$ is of the order of the finite (pure phase) correlation lengths $\xi_{o, d}$ in the ordered and disordered phase, and from contributions $\propto \exp \left(-2 \sigma_{o d} L^{D-1}\right)$ of the two-phase region, where $\sigma_{o d}$ is the (reduced) interface tension between the two phases. For $3 \mathrm{D}$ systems the latter correction decays asymptotically much faster and, in general, both types of exponential corrections are always weaker than the power-law terms for large $L$. For relatively small system sizes with $L$ of the order of $\xi_{o, d}$ or $\sigma_{o d}^{-1 /(D-1)}$, however, it is a priori not clear which type of scaling behaviour will dominate. A possible explanation for the observed dip in the scaling behaviour of the 3D 3-state Potts model is therefore that for $L<30$ the exponential terms dominate and then rapidly die out, giving way to an 
inverse volume dependence for $L>30$.

Consequently, in order to extract the infinite-volume transition point, $\beta_{t}$, from a pure power-law ansatz in the inverse volume, in previous studies only the data from lattices with $L \geq 30$ have been taken into account. Of course, this is an expensive task because the computer time to generate accurate enough data points for such lattice sizes becomes exceedingly large, even with refined MC techniques for first-order phase transitions such as multicanonical [30] or multibondic [31] algorithms which reduce autocorrelation times significantly.

Recently a new set of observables was proposed which do not show any power-law terms in their FSS behaviour [28, 29]. It is thus governed only by exponentially small terms. For lattices with periodic boundary conditions and large enough $q$, this could be proven exactly [25]. In Refs. [28, 29] the new set of observables was first used to study the first-order phase transitions of the two-dimensional $q$-state Potts model with $q=5,8$, and 10 . It was found that the new methods yield surprisingly accurate results already for very small lattice sizes and even for the extremely weak first-order transition of the 2D 5-state model. Since this might be a fortuitous accident of the 2D model we found it worthwhile to explore the accuracy of the new methods in the 3D case as well. The purpose of this paper is thus to test the validity of the new observables for three-dimensional lattices and for a $q$ value as small as 3. We shall see that, due to the absence of power-law corrections, the dip in the new pseudo-transition points $\beta_{t}(L)$ indeed disappears, and a good fit to extract the infinite-volume transition point $\beta_{t} \equiv \beta_{t}(\infty)$ becomes feasible without the need of extremely large lattices.

The rest of the paper is organized as follows. In Sec. 2 we recall the definition of the model and briefly summarize previous results, and in Sec. 3 we recapitulate the definition of the new observables. The results of our analysis are presented in Sec. 4, and in Sec. 5 we conclude with a brief discussion.

\section{Model and previous results}

We use the standard definition of the 3-state Potts model [1],

$$
Z=\sum_{\left\{\sigma_{i}\right\}} e^{-\beta E}=\sum_{E} N(E) e^{-\beta E}, \quad E=-\sum_{\langle i j\rangle} \delta_{\sigma_{i}, \sigma_{j}}, \quad \sigma_{i}=1,2,3,
$$


where $\beta=J / k_{\mathrm{B}} T$ is the inverse temperature in natural units, $N(E)$ is the number of configurations with energy $E,\langle i j\rangle$ indicates that the sum runs over nearest-neighbour pairs, and $\delta_{\sigma_{i}, \sigma_{j}}$ is the Kronecker symbol. The energy per site is denoted by $e=E / V$, where $V=L^{3}$ is the volume of the system. The data we use to test our observables was obtained in a previous MC simulation by Alves et al. (ABV) [12] who employed heat-bath updating for lattices of size $L=10,12,14,18,22,24,30$, and 36 , with periodic boundary conditions. For all the lattice sizes, the simulations were performed at $\beta_{\mathrm{MC}}=0.55059$, with $50 \times 10^{6}$ energy measurements for $L=10$, 12, and 14 , and $20 \times 10^{6}$ measurements for the larger lattice sizes.

By means of standard reweighting procedures [32], the data was used to compute the maximum $C_{\max }=C\left(\beta_{C_{\max }}\right)$ of the specific heat, $C(\beta)=$ $\beta^{2} V\left(\left\langle e^{2}\right\rangle-\langle e\rangle^{2}\right)$, and the Fisher zeros of the partition function. Finite-size scaling was finally applied to both the specific-heat maxima and the partition function zeros in order to extract the transition point and the FSS exponents. Due to the above described problem with the non-monotonic scaling behaviour of $\beta_{C_{\max }}(L)$, ABV combined their data with those of Ref. [11] on larger lattices and extracted their final estimate ${ }^{1}$ of

$$
\beta_{t}=0.550523 \pm 0.000010 \quad(\mathrm{ABV})
$$

from a power-law fit, $\beta_{C_{\max }}=\beta_{t}+c / V$, using only the largest lattices of size $L=30,36,42$, and 48 . The latent heat,

$$
\Delta e \equiv e_{d}-e_{o}=0.16062 \pm 0.00052 \quad(\mathrm{ABV})
$$

was obtained from the FSS of the specific-heat maxima on smaller lattices of size $L=22,24,30$, and 36, since the scaling behaviour of $C_{\max }$ turned out to be better behaved than that of the maxima locations. An overview of other previous estimates of $\beta_{t}$ is given in Table 1 .

\section{The new observables}

The definition of the new observables is based on the observation made in Ref. [25] that for a $q$-state Potts model on a lattice with periodic boundary

\footnotetext{
${ }^{1}$ The notation of ABV follows the high-energy physics conventions. The translation into the present notation, usually used in condensed matter physics, reads $\beta_{t}=(3 / 2) \beta_{t}^{(\mathrm{ABV})}$. Similarly the latent heats are related by $\Delta e=2 \Delta e^{(\mathrm{ABV})} \equiv 2 \ell$.
} 
Table 1: The transition point $\beta_{t}$ of the 3-state Potts model on the simple cubic lattice in units $k_{\mathrm{B}}=1=J$

\begin{tabular}{ll}
\hline \multicolumn{1}{c}{$\beta_{\mathrm{t}}$} & \multicolumn{1}{c}{ author(s) } \\
\hline 0.5348 & Straley (1974) $[2]$ \\
$0.5596(54)$ & Kim and Joseph (1975) [3] \\
$0.54749(52)$ & Miyashita et al. (1979) [4] \\
0.550 & Blöte and Swendsen (1979) [5] \\
$0.55006(91)$ & Herrmann (1979) [6] \\
$0.55040(19)$ & Knak-Jensen and Mouritsen (1979) [7] \\
0.552 & Ono and Ito (1982) [8] \\
$0.55059(2)$ & Wilson and Vause (1987) [9] \\
$0.55062(3)$ & Gavai et al. (1989) [10] \\
$0.550545(45)$ & Fukugita et al. (1990) [11] \\
$0.550523(10)$ & Alves et al. (1991) [12] \\
$0.550479(61)$ & Yamagata (1993) [13] \\
$0.550565(10)$ & this work \\
\hline
\end{tabular}

conditions, provided $q$ is large enough, the partition function can be written as

$$
Z_{\text {per }}(V, \beta)=\left[\sum_{m=0}^{q} e^{-\beta f_{m}(\beta) V}\right]\left[1+O\left(V e^{-L / L_{0}}\right)\right],
$$

where $L_{0}<\infty$ is a constant, $L$ is the linear length of the lattice, and $f_{m}(\beta)$ is the metastable free-energy density of the phase $m$, where $m=0$ is associated with the disordered and $m=1, \ldots, q$ with the $q$ ordered phases. It can be defined in such a way that it is equal to the idealized infinite-volume free-energy density $f(\beta)$ if $m$ is stable and strictly larger than $f(\beta)$ if $m$ is unstable. At $\beta_{t}$ all $f_{m}$ are equal.

In Refs. [28, 29] it is discussed how Eq. (2) leads to a definition of pseudotransition points $\beta_{V / V}$ for finite systems, which deviate from the infinitevolume transition point $\beta_{t}$ only by exponentially small terms. These points are obtained by locating the maximum of the number-of-phases observable

$$
N\left(V, V^{\prime}, \beta\right) \equiv\left[\frac{Z_{\mathrm{per}}(V, \beta)^{\alpha}}{Z_{\mathrm{per}}\left(V^{\prime}, \beta\right)}\right]^{1 /(\alpha-1)},
$$


where $\alpha=V^{\prime} / V$. By inserting (2) it is easy to see that $N\left(V, V^{\prime}, \beta\right)$ indeed counts the number of phases: $q$ at low temperatures, $q+1$ at phase coexistence, and 1 in the disordered high-temperature phase. Searching for the maximum of $N\left(V, V^{\prime}, \beta\right)$ as a function of $\beta$ amounts to locating the crossing points of the internal energies per site for the two lattices of different size,

$$
e\left(V, \beta_{V / V^{\prime}}\right)=e\left(V^{\prime}, \beta_{V / V^{\prime}}\right) .
$$

As a consequence of (2) it can be proven that, for large enough $q$, the crossing points $\beta_{V / V^{\prime}}$ are only exponentially shifted from the infinite-volume transition

point $\beta_{t}$. In contrast to the pseudo-transition points $\beta_{C_{\max }}(L)$, there are no powers of the inverse volume involved.

Also in Refs. [28, 29], yet another definition of pseudo-transition points, $\beta_{w}(L)$, without power-law corrections was proposed which are obtained by measuring the ratio-of-weights,

$$
R_{W}(V, \beta) \equiv \frac{W_{o}}{W_{d}} \equiv \sum_{E<E_{0}} P_{\beta}(E) / \sum_{E \geq E_{0}} P_{\beta}(E),
$$

and solving the equation

$$
\left.R_{W}(V, \beta)\right|_{\beta=\beta_{w}}=q=3 .
$$

Here, $P_{\beta}(E)$ stands for the probability distribution $P_{\beta}(E)=N(E) e^{-\beta E} / Z$, with $Z$ and $N(E)$ defined in Eq. (1). The parameter $E_{0}$ in Eq. (5) is defined by reweighting the probability distribution to the point $\beta_{P}$ where the two peaks of $P_{\beta}(E)$ have equal height and then taking $E_{0}$ as the energy where $P_{\beta_{P}}(E)$ has the minimum between the two peaks. This defines still another sequence of pseudo-transition points $\beta_{P}$ which, however, is not expected to exhibit a FSS behaviour free of $1 / V$ powers.

\section{Results}

\subsection{Number-of-phases criterion}

From Eq. (4) it is clear that the numerical determination of $\beta_{V / V^{\prime}}$ requires handling simultaneously two different lattices sizes. Once they are chosen, searching for the minimum of

$$
\left|e\left(V, \beta_{V / V^{\prime}}\right)-e\left(V^{\prime}, \beta_{V / V^{\prime}}\right)\right|=0,
$$


Table 2: Pseudo-transition points $\beta_{V / V^{\prime}}$ for pairs of lattices of size $L$ and $L^{\prime}$

\begin{tabular}{l|cccccc}
$L \backslash L^{\prime}$ & 14 & 18 & 22 & 24 & 30 & 36 \\
\hline 10 & $0.552309(24)$ & $0.551757(15)$ & $0.551421(15)$ & $0.5513025(75)$ & $0.5510784(59)$ & $0.5509544(50)$ \\
12 & $0.551798(41)$ & $0.551405(21)$ & $0.551142(13)$ & $0.5510459(72)$ & $0.5508701(48)$ & $0.5507715(56)$ \\
14 & & $0.551220(30)$ & $0.550998(17)$ & $0.5509160(95)$ & $0.5507778(53)$ & $0.5507039(57)$ \\
18 & & & $0.550808(41)$ & $0.550751(18)$ & $0.5506733(90)$ & $0.5506343(63)$ \\
22 & & & & $0.550649(66)$ & $0.550624(15)$ & $0.5506043(87)$ \\
24 & & & & & $0.550618(14)$ & $0.5505995(83)$ \\
30 & & & & & & $0.550586(18)$
\end{tabular}

using standard numerical minimizing routines combined with reweighting procedures, leads to the $\beta_{V / V^{\prime}}$ collected in Table 2 for all possible pairs of sizes $L$ and $L^{\prime}$. Here and in the following statistical error bars are estimated by means of the jack-knife method [33] on the basis of 20 blocks.

The question arises on how to extract an infinite-volume transition point from the results given in Table 2. Considering that, as discussed in Sec. 3, the pseudo-transition points $\beta_{V / V^{\prime}}$ are left with only exponential deviations from $\beta_{t}$, we first tried a fit of the form

$$
\beta_{V / V^{\prime}}(L)=\beta_{t}+a_{1} e^{-b_{1} L},
$$

with $L^{\prime}>L$ kept fixed. It seems evident that the bigger $V$ and $V^{\prime}$ the better the fit. By keeping $L^{\prime}=36$ fixed and using $L=12, \ldots, 30$ (i.e., the last column in Table 2 without the $L=10$ entry) we obtain an estimate of

$$
\beta_{t}=0.550586 \pm 0.000010 \quad \text { (number-of-phases criterion), }
$$

with $a_{1}=0.0027(10), b_{1}=0.224(34)$, and a goodness-of-fit parameter of $Q=0.99$ (corresponding to a total chi-squared of $\chi^{2}=0.080$ with 3 degrees of freedom (dof)). The goodness of the fit can be visually inspected in Fig. 1. Strictly speaking the parameters in (8) should still depend on the (larger) lattice size $L^{\prime}$. To estimate this effect we performed a similar fit with $L^{\prime}=30$ fixed, using the crossing points with the smaller lattices of size $L=12, \ldots, 24$. As a result we obtained a consistent estimate of $\beta_{t}=0.550590(20), a_{1}=$ $0.00312(89), b_{1}=0.201(28)$, and $Q=0.95$. 
As far as the systematic FSS corrections are concerned it would be more reasonable to extrapolate the $\beta_{V / V^{\prime}}$ along the diagonal of Table 2 , where $L^{\prime} / L \approx 1.1 \ldots 1.3$, such that both, $L$ and $L^{\prime}$, are sent to infinity simultaneously. From the point of statistical errors, however, this procedure is somewhat problematic. First, the crossing points of energies for only slightly different lattice sizes have naturally the biggest errors since the slopes $d e(V, \beta) / d \beta$ and $d e\left(V^{\prime}, \beta\right) / d \beta$ differ only little. This is clearly reflected in Table 2 . Second, and more difficult to take properly into account in principle, the data for, e.g., $\beta_{12^{3} / 14^{3}}$ and $\beta_{14^{3} / 18^{3}}$ are correlated since both involve the energy on the $14^{3}$ lattice. To avoid this correlation one would have to simulate many more different lattice sizes. We nevertheless also tried this type of fit and, using all 6 data points along the diagonal (with $L$ in (8) being the smaller of the two lattice sizes), obtained $\beta_{t}=0.550586(15), a_{1}=0.048(16)$, and $b_{1}=0.307(26)$, with $Q=0.79$. It is gratifying that the extrapolated values of $\beta_{t}$ are in perfect agreement for all three sequences of pseudo-transition points, even though the approach to the infinite-volume limit shown in Fig. 1 looks quite different, in particular for the last sequence along the diagonal.

\subsection{Ratio-of-weights criterion}

From a technical point of view, the second new definition (6) of pseudotransition points, $\beta_{w}$, is probably somewhat easier to evaluate numerically since it involves only one lattice size at a time. A slight complication arises, however, from the fact that one has first to estimate $E_{0}$, the energy cut separating the ordered from the disordered phase. In order to determine $E_{0}$, we proceeded as follows. For a given lattice size $L$, we reweight the probability distribution of the energy to the point $\beta_{P}$ where the ordered and disordered peaks are of equal height,

$$
P_{1, \max }=P_{\beta_{P}}\left(E_{1, \max }\right)=P_{\beta_{P}}\left(E_{2, \max }\right)=P_{2, \max } .
$$

We then fix $\beta$ to $\beta_{P}$ and search for the minimum of $P_{\beta_{P}}(E)$ for $E$ 's satisfying $E_{1, \max }<E<E_{2, \max }$. Our $E_{0}$ is thus defined by $P_{\min }=P_{\beta_{P}}\left(E_{0}\right)$. Once $E_{0}$ is determined it is kept fixed, and we reweight again over $\beta$ until Eq. (6) is solved for $\beta=\beta_{w}$. The behaviour of $R_{W}(V, \beta)$ as a function of $\beta$ is shown in Fig. 2.

The Table 3 collects the $\beta_{P}$ and $\beta_{w}$ introduced in Eqs. (10) and (6) for $L=$ $10, \ldots, 36$. Also given are the values of $P_{\min }$, employing the normalization 
Table 3: Table of $\beta_{P}, P_{\min }$ and the corresponding $2 \sigma_{o d}$, together with $\beta_{w}$

\begin{tabular}{c|lccl}
$L$ & \multicolumn{1}{|c}{$\beta_{P}$} & $P_{\min }$ & \multicolumn{1}{c}{$2 \sigma_{o d}$} & \multicolumn{1}{c}{$\beta_{w}$} \\
\hline 10 & $0.550633(12)$ & $0.8576(26)$ & $0.001536(30)$ & $0.552223(10)$ \\
12 & $0.550638(20)$ & $0.7911(34)$ & $0.001627(30)$ & $0.5516148(72)$ \\
14 & $0.550585(11)$ & $0.7310(43)$ & $0.001599(30)$ & $0.5512068(62)$ \\
18 & $0.550572(15)$ & $0.5869(81)$ & $0.001645(43)$ & $0.550878(10)$ \\
22 & $0.550567(12)$ & $0.4561(87)$ & $0.001622(39)$ & $0.550700(10)$ \\
24 & $0.5505521(77)$ & $0.3984(94)$ & $0.001598(41)$ & $0.5506586(65)$ \\
30 & $0.5505560(65)$ & $0.2291(54)$ & $0.001638(26)$ & $0.5505965(50)$ \\
36 & $0.5505665(72)$ & $0.1155(66)$ & $0.001666(44)$ & $0.5505812(59)$
\end{tabular}

$P_{1, \max }=P_{2, \max }=1$, as well as

$$
2 \sigma_{o d}(L)=-\ln \left(P_{\min }\right) / L^{2},
$$

which serves as a finite-volume estimator of the (reduced) interface tension between the ordered and disordered phase [34] to be discussed below.

In order to extract an estimate for the infinite-volume transition point, we tried again an exponential fit of the form

$$
\beta_{w}(L)=\beta_{t}+a_{2} e^{-b_{2} L} .
$$

Using the $\beta_{w}(L)$ for $L=14, \ldots, 36$ from Table 3 , we extract

$$
\beta_{t}=0.5505681 \pm 0.0000056 \quad \text { (ratio-of-weights criterion), }
$$

with $a_{2}=0.00939(93), b_{2}=0.1919(74)$, and $Q=0.41$. The data for $\beta_{w}$ and $\beta_{P}$ together with the fit (12) are shown in Fig. 3. Within error bars the estimates (9) and (13) are compatible with each other, but both are slightly higher than the previous estimate of ABV. Notice that also the estimates $b_{1}$ and $b_{2}$ are consistent, indicating that $L_{0}=1 / b_{1,2}$ in (2) is about $L_{0} \approx 5$. This value is roughly one half of the correlation length estimates $\xi_{o} \approx \xi_{d} \approx 10.2$, obtained recently in pure phase simulations of the 3 -state Potts model on a $100^{3}$ lattice [35]. 
In general also the pseudo-transition points $\beta_{P}$ (where the two peaks are of equal height) can be used to extract $\beta_{t}$. As mentioned at the end of Sec. 3, the FSS behaviour should be qualitatively similar to $\beta_{C_{\max }}$, i.e., one expects an asymptotic power series in $1 / V$. A glance at Fig. 3 shows, however, that the $\beta_{P}$ for the three-dimensional 3-state Potts model are apparently almost constant. In fact, by simply taking the weighted average of the six values for $L=14, \ldots, 36$ (i.e., performing a trivial fit $\beta_{P}=\beta_{t}=$ const.), we obtain an estimate of

$$
\beta_{t}=0.5505625 \pm 0.0000036 \quad \text { (equal-peak-height locations) }
$$

with $Q=0.16$, in good agreement with (13). If we omit the $L=14$ value, the average changes only little to $\beta_{t}=0.5505600(38)$, with $Q=0.51$.

This completely unexpected result can be understood as follows: The decomposition (2) of the partition function translates to the probability distribution (formally via an inverse Laplace transformation) at $\beta_{t}$ in a Gaussian approximation to

$$
\begin{aligned}
P_{\beta_{t}}(e) & =q \sqrt{\frac{V \beta_{t}^{2}}{2 \pi c_{o}}} \exp \left[-\frac{\left(e-e_{o}\right)^{2} \beta_{t} V}{2 c_{o}}\right]+\sqrt{\frac{V \beta_{t}^{2}}{2 \pi c_{d}}} \exp \left[-\frac{\left(e-e_{d}\right)^{2} \beta_{t} V}{2 c_{d}}\right] \\
& \equiv H_{o}(e)+H_{d}(e),
\end{aligned}
$$

where $e_{o}\left(e_{d}\right)$ and $c_{o}\left(c_{d}\right)$ are the energy and specific heat in the ordered (disordered) phase at $\beta_{t}$, and $H_{o}\left(H_{d}\right)$ approximates the "ordered" ("disordered") part of the measured histograms. ${ }^{2}$ This implies for the ratio of heights at $\beta_{t}$

$$
R_{H}\left(\beta_{t}\right) \equiv \frac{H_{o}^{\max }}{H_{d}^{\max }}=q \sqrt{\frac{c_{d}}{c_{o}}} .
$$

Estimates of $c_{o}$ and $c_{d}$ have recently been obtained in Ref. [35] from simulations of a $100^{3}$ lattice in the pure phases at $\beta=0.55053$, the previously best estimate of $\beta_{t}$. If we perform a slight reweighting to our present best estimate of $\beta_{t}=0.550565$ (see the Conclusions below) and insert the resulting numbers $\left(c_{o}=28.84, c_{d}=3.210\right)$ into (16) we obtain

$$
R_{H}\left(\beta_{t}\right)=1.001(18)
$$

\footnotetext{
${ }^{2}$ In practice the separation of a given histogram into $H_{o}$ and $H_{d}$ is of course not unique. Using as dividing energy the cut $E_{0}$ introduced earlier, then $\sum_{e} H_{o}(e)=W_{o}$ and $\sum_{e} H_{d}(e)=W_{d}$. The Gaussian approximation is therefore a good approximation in the sense that $W_{o} / W_{d}=q$ at $\beta_{t}$.
} 
where the error emerges from the statistical uncertainties in $c_{o}$ and $c_{d}$. The error due to the uncertainty in $\beta_{t}$ is of the same order; see Fig. 4 , where $R_{H}(\beta)$ is plotted over a range of $\beta$ values around $\beta_{t}$. We thus have the surprising result that for the three-dimensional 3-state Potts model the first-order transition point $\beta_{t}$ is not only characterized by the, on quite general grounds, theoretically predicted ratio-of-weights $R_{W}\left(\beta_{t}\right)=q=3$, but empirically also by an equal-peak-height condition, $R_{H}\left(\beta_{t}\right)=1$. This is qualitatively the reason why $\beta_{P}$ happens to be so close to the infinite-volume transition point $\beta_{t}$. The actually measured probability distributions for $L=36$ reweighted to (a) $\beta=\beta_{w}(L=36)=0.5505812$ and (b) $\beta=\beta_{p}(L=36)=0.5505665$ are shown in Fig. 5 . Notice the sensitivity to small variations in $\beta$ of the order of $2-3$ error bars of $\beta_{w}$ or $\beta_{p}$.

The result $c_{o} / c_{d} \approx 9=q^{2}$ is presumably an accidental peculiarity of this particular 3D 3-state Potts model. For $2 \mathrm{D} q$-state Potts models with $q \geq 5$ it is exactly known that $c_{o} \approx c_{d}$ at $\beta_{t}$, such that $R_{H}\left(\beta_{t}\right) \approx q$, and also for the $3 \mathrm{D} q$-state models with $q=4$ and 5 one finds values of $R_{H}\left(\beta_{t}\right) \approx 1.4$ $(q=4)$ and $R_{H}\left(\beta_{t}\right) \approx 1.8(q=5)$ (using again the estimates for $c_{o}$ and $c_{d}$ of Ref. [35]) which clearly deviate from unity. On the other hand, in (17) unity is hit so accurately that it is tempting to speculate that there could be some hidden theoretical reason for this numerical observation (possibly related to the $Z_{3}$ symmetry of the 3 -state Potts model). It would be very interesting to test this possibility by studying the 3-state model on other three-dimensional lattices $(\mathrm{BCC}, \mathrm{FCC}, \ldots)$ or in higher dimensions $(4 \mathrm{D}, 5 \mathrm{D}, \ldots)$.

\subsection{Locations of $C_{\max }$}

Next we also reconsider the scaling of the locations $\beta_{C_{\max }}$ of the specificheat maxima, showing the peculiar dip around $L=30$. As discussed in the Introduction a possible explanation is a crossover between exponential corrections $\propto \exp \left(-L / L_{0}\right)$ and the asymptotic power series in $1 / V$. We therefore tried to fit the data with an ansatz

$$
\beta_{C_{\max }}(L)=\beta_{t}+\frac{c}{V}+a_{3} e^{-b_{3} L} .
$$

By following the procedure of ABV and including also the data of Ref. [11], but discarding only the three points with $L \leq 14$, we obtained

$$
\beta_{t}=0.550552 \pm 0.000020 \quad\left(C_{\max } \text { locations for } L \leq 48\right),
$$


$c=-4.1(1.3), a_{3}=0.0309(34)$, and $b_{3}=0.207(16)$, with $Q=0.46$. The fit is shown as the dashed line on the fine scale of Fig. 6. By including also the $L=14$ data in the fit, the numbers change only slightly to $\beta_{t}=0.550535(12)$, $c=-2.86(59), a_{3}=0.0356(25)$, and $b_{3}=0.2324(82)$, with $Q=0.41 .^{3}$

One interesting question was if now already the data of ABV up to $L=36$ would be sufficient to get a reliable estimate of $\beta_{t}$. Using their five data points for $L=18, \ldots, 36$, we indeed obtained a reasonable fit with

$$
\beta_{t}=0.550569 \pm 0.000042 \quad\left(C_{\max } \text { locations for } L \leq 36\right),
$$

$c=-4.9(2.6), a_{3}=0.0351(53), b_{3}=0.207(21)$, and $Q=0.71$. As can be inspected in Fig. 6 the two fits are in fact hardly distinguishable.

As a consequence of the additional exponential term in the fit ansatz (18) the estimates of $\beta_{t}$ in (19) and (20) are somewhat higher than the result of ABV obtained from a pure power-law ansatz. In particular the estimate (20) relying only on the data of ABV is now in perfect agreement with the results from the new methods discussed above. It appears quite natural that the mixing with the data of Ref. [11] perturbs this self-consistency a little bit.

Even though the high values of $Q$ indicate that both fits are statistically self-consistent, it is a priori clear that (18) can only be an effective model of the true behaviour, since higher order terms in $1 / V$ are neglected. A simple way to test for this is to compare the coefficient $c$ of the $1 / V$ term with the prediction of FSS theory $[29,36], c=-\ln q / \Delta e \approx-6.5$, where $\Delta e$ can be taken from ABV or from the analysis in the next section. We see that within the relatively large error bars the fitted values of $c$ are compatible with this expectation, but there is definitely a trend to underestimate $c$. We have checked that the pure power-law fit used by ABV, $\beta_{C_{\max }}=\beta_{t}+c / V$, has the same problem; we can reproduce their $\beta_{t}$ to all digits and obtain $c=-1.77(33)$. This shows that the additional exponential correction helps at least to drive $c$ in the right direction. A glance at Fig. 6 then shows that a pure power-law fit must necessarily underestimate $\beta_{t}$.

How about higher-order corrections in the $1 / V$ expansion? The FSS

\footnotetext{
${ }^{3}$ It should be noted, however, that the ansatz (18) admits also another fit with "unreasonable" parameters $\left(c>0\right.$ and $\left.a_{3}<0\right)$ which actually has even a slightly better $Q$ value of $Q=0.43$.
} 
expansion in $1 / V$ up to second order reads $[29,36]$

$$
\beta_{C_{\max }}=\beta_{t}-\frac{\beta_{t} \ln q}{V \Delta s}+\frac{\beta_{t}}{(V \Delta s)^{2}}\left[4-6 \frac{\Delta c}{\Delta s}+\frac{\Delta c}{2 \Delta s}(\ln q)^{2}\right]+\mathcal{O}\left(1 / V^{3}\right),
$$

where $\Delta s=\beta_{t} \Delta e$ is the entropy jump, $\Delta e=e_{d}-e_{o}$ the latent heat, $\Delta c=$ $c_{d}-c_{o}$ the specific-heat jump, and $e_{o}, c_{o}$ etc. are to be taken as the (pure phase) expectation values at $\beta_{t}$. Inserting $\beta_{t}=0.550565$ and again the estimates of Ref. [35], we obtain the explicit FSS prediction

$$
\beta_{C_{\max }}=0.550565-6.50255 / V+94804.0 / V^{2}+\ldots
$$

In Fig. 7 the resulting first- and second-order FSS scaling curves are displayed as the solid lines on the same scale as in Fig. 6. As a rough error estimate the dashed lines show Eq. (21) evaluated at the error bounds of $\beta_{t}$, i.e., at $0.550565 \pm 0.000010$. The statistical error coming from the uncertainties in the pure phase expectation values is of the same order. At first sight one is inclined to conclude that the second-order FSS curve tends to reproduce the dip in the data. At its minimum, however, the difference between the FSS expansions to first and second order is already quite large, and one may suspect that the omitted terms of $\mathcal{O}\left(1 / V^{3}\right)$ would change the picture drastically. In fact, by extending the expansion of $\beta_{C_{\max }}$ up to the fourth order in $1 / V$ [37] we clearly observe the breakdown of this asymptotic expansion for moderate values of $V$. A similar observation was recently made in Ref. [38] for the maxima locations of $C / \beta^{2}=V\left(\left\langle e^{2}\right\rangle-\langle e\rangle^{2}\right)$ in the two-dimensional 10 -state Potts model, using large- $q$ expansions to estimate the various energy cumulants involved.

\subsection{Latent heat and interface tension}

As proposed in Ref. [29] the ratio-of-weights method provides at $\beta_{t}$ also a natural estimator for the latent heat,

$$
\Delta e(L)=\frac{d}{d \beta} \ln \left(W_{o} / W_{d}\right) / V
$$

which, similar to $\beta_{w}(L)$, should show only exponential deviations from the infinite-volume limit, $\Delta e(\infty)=e_{d}-e_{o}$. Graphically, the $V \Delta e(L)$ are just the slopes of $\ln R_{W}(\beta)=\ln \left(W_{o} / W_{d}\right)$ shown in Fig. 2 which can, of course, be 
Table 4: Finite lattice estimates for the latent heat using the ratio-of-weights method (with $\beta_{w}(\infty)=0.5505681$ )

\begin{tabular}{c|ll}
$L$ & $\Delta e$ at $\beta_{w}(\infty)$ & $\Delta e$ at $\beta_{w}(L)$ \\
\hline 10 & $0.28650(12)$ & $0.30404(12)$ \\
12 & $0.25045(13)$ & $0.26462(14)$ \\
14 & $0.22576(10)$ & $0.23666(10)$ \\
18 & $0.19549(23)$ & $0.20297(23)$ \\
22 & $0.17926(28)$ & $0.18380(28)$ \\
24 & $0.17392(23)$ & $0.17739(23)$ \\
30 & $0.16580(30)$ & $0.16734(30)$ \\
36 & $0.16343(37)$ & $0.16433(36)$
\end{tabular}

computed in precisely this way by numerical differentiation. It is, however, more convenient and numerically more stable to perform the differentiation in (23) explicitly. Recalling the definition (5) it is easy to see that

$$
\Delta e(L)=\langle e\rangle_{d}-\langle e\rangle_{o},
$$

where $\langle e\rangle_{d} \equiv \frac{1}{V} \sum_{E \geq E_{0}} E P_{\beta}(E) / \sum_{E \geq E_{0}} P_{\beta}(E)$ is the expectation value of the energy computed over the disordered peak only, and $\langle e\rangle_{o}$ is defined analogously. We computed $\Delta e(L)$ in two different ways. First, at the infinitevolume limit of $\beta_{w}(L), \beta=0.5505681 \approx \beta_{t}$, and second self-consistently at the lattice size dependent sequence of $\beta=\beta_{w}(L)$. The resulting data is given in Table 4 and shown in Fig. 8.

The fits in Fig. 8 are according to the ansatz

$$
\Delta e(L)=\Delta e+a_{4} e^{-b_{4} L},
$$

where $\Delta e \equiv \Delta e(\infty)$ is the infinite-volume limit of the latent heat. When $\beta=0.5505681 \approx \beta_{t}$ is held fixed, $\Delta e(L)$ follows the exponential ansatz down to quite small lattice sizes, and by discarding only the $L=10$ data point we obtain the infinite-volume estimate

$$
\Delta e=0.16121 \pm 0.00027,
$$

$a_{4}=0.6160(80)$, and $b_{4}=0.1611(12)$, with $Q=0.14$. When the $\Delta e(L)$ are evaluated at $\beta_{w}(L)$, the asymptotic scaling region is shifted to larger values 
of $L$. In order to get a fit of reasonable quality, here we had to discard the data for $L \leq 14$. The result of the fit,

$$
\Delta e=0.16160 \pm 0.00047
$$

with $a_{4}=0.733(50), b_{4}=0.1597(42)$, and $Q=0.11$, is then compatible with (26), and both estimates are consistent with the previous estimate of ABV from the scaling of the specific-heat maxima.

Let us finally consider the (reduced) interface tension $\sigma_{o d}$. As can be read off from Table 3 , the data for $L=12, \ldots, 36$ vary so little that none of the more sophisticated FSS extrapolations is applicable. Similar to the analysis of $\beta_{p}$ we therefore have again simply taken a weighted average of the seven values with the result

$$
2 \sigma_{\text {od }}=0.00163 \pm 0.00002
$$

and $Q=0.87$. A similar estimate of $2 \sigma_{o d}=0.00157(6)$ was recently obtained in Ref. [22].

\section{Conclusions}

In this paper we have presented a detailed study of the first-order phase transition in the three-dimensional 3-state Potts model on cubic lattices with emphasis on recently introduced quantities whose infinite-volume extrapolations are governed only by exponentially small terms. The main results are the following:

(i) The phase transition is only weakly first-order. Nevertheless the new techniques, originally introduced for "large enough" $q$, i.e., strong first-order phase transitions, prove to be surprisingly accurate. The expected exponential finite-size scaling of the pseudo-transition points $\beta_{V / V^{\prime}}$ and $\beta_{w}$ as well as of the "ratio-of-weights" definition of the latent heat is clearly observed. The results of fits of the form $\beta_{t}(L)=\beta_{t}+a \exp (-b L)$ to the various sequences of pseudo-transition points are summarized in Table 5. Taking into account the small remaining systematic uncertainties by averaging over the entries

in Table 5 we quote as our final estimate for the infinite-volume transition point

$$
\beta_{t}=0.550565 \pm 0.000010 \text {. }
$$


Table 5: Summary of our various estimates of $\beta_{t}$

\begin{tabular}{|l|l|l|l|c|}
\hline obs. & \multicolumn{1}{|c|}{ range } & \multicolumn{1}{c|}{ ansatz } & \multicolumn{1}{c|}{$\beta_{t}$} & $Q$ \\
\hline$\beta_{V / V^{\prime}}$ & $L^{\prime}=36, L=12-30$ & $\beta_{t}+a e^{-b L}$ & $0.550586(10)$ & 0.99 \\
$\beta_{V / V^{\prime}}$ & $L^{\prime}=30, L=12-24$ & $\beta_{t}+a e^{-b L}$ & $0.550590(20)$ & 0.95 \\
$\beta_{V / V^{\prime}}$ & diagonal & $\beta_{t}+a e^{-b L}$ & $0.550586(15)$ & 0.79 \\
\hline$\beta_{w}$ & $L=14-36$ & $\beta_{t}+a e^{-b L}$ & $0.5505681(56)$ & 0.41 \\
\hline$\beta_{P}$ & $L=14-36$ & average & $0.5505625(36)$ & 0.16 \\
\hline$\beta_{C_{\max }}$ & $L=14-48$ & $\beta_{t}+c / V+a e^{-b L}$ & $0.550535(12)$ & 0.41 \\
$\beta_{C_{\max }}$ & $L=18-48$ & $\beta_{t}+c / V+a e^{-b L}$ & $0.550552(20)$ & 0.46 \\
$\beta_{C_{\max }}$ & $L=18-36$ & $\beta_{t}+c / V+a e^{-b L}$ & $0.550569(42)$ & 0.71 \\
\hline
\end{tabular}

(ii) The points $\beta_{p}$, where the two peaks of the energy probability distribution are of equal height, show surprisingly almost no finite-size scaling. This in turn implies that in this particular model the transition point is not only characterized by the theoretically founded "3:1-weight rule", but empirically to a very good approximation also by an "equal-height rule". We emphasize that this could be a purely accidental situation since an "equal-height rule" is definitely not satisfied in two-dimensional $q$-state Potts models for all $q \geq 5$ and in the three-dimensional models with $q=4$ and 5 .

(iii) The peculiar dip in the finite-size scaling of the locations of the specific-heat maxima can be explained by a fit of the form $\beta_{C_{\max }}(L)=c / V+$ $a \exp (-b L)$.

(iv) The decay constant $b$ in the exponential term $\exp (-b L)$ is quite consistently found from the various fits around $b \approx 0.2$. This implies $L_{0}=$ $1 / b \approx 5$, which is about one-half of the correlation length $\xi_{d} \approx \xi_{o} \approx 10$ in the pure disordered and ordered phases. A similar relation was observed in Refs. [40,41] for the two-dimensional 10-state Potts model, where $\xi_{d}$ is known exactly and evidence for $\xi_{o}=\xi_{d}$ was obtained numerically with high accuracy [42].

(v) Similar to the discussion of the two-dimensional 10-state Potts model 
in Ref. [38], we find that the asymptotic expansion in $1 / V$ for $\beta_{C_{\max }}$ is illbehaved. Only for very large system sizes higher order terms lead to an improvement which, however, is quite a general feature of asymptotic expansions.

(vi) The finite-size scaling of the "ratio-of-weights" definition of the latent heat follows the predicted exponential behaviour. The resulting infinitevolume estimate,

$$
\Delta e=0.1614 \pm 0.0003
$$

was found compatible with the previous estimate of ABV from the finite-size scaling of the specific-heat maxima.

(vii) The interface tension $\sigma_{\text {od }}$ between the ordered and disordered phases has probably not yet reached its asymptotic scaling region for lattice sizes up to $L=36$. Experience with three-dimensional $q$-state Potts models with $q=4$ and 5 suggests [43] that our estimate $2 \sigma_{o d}=0.00163(2)$ is a lower bound on the infinite-volume limit. Still it is difficult to reconcile this value with the estimates of $\sigma_{o o}\left(\beta_{t}\right) \geq 0.0048(3)$ and $\sigma_{o o}\left(\beta_{t}\right)=0.0040(7)$ in Ref. [17] and the quite general stability condition $\sigma_{o o}\left(\beta_{t}\right) \leq 2 \sigma_{o d}$ [44]. Notice that at least in two dimensions equality seems to hold in the last relation ("complete wetting").

\section{Acknowledgements}

We would like to thank Stefan Kappler for many useful discussions. This work was supported in part by a collaborative research grant within the Programme "Acciones Integradas" of the DAAD/Spanish Ministry of Education. WJ thanks the Deutsche Forschungsgemeinschaft (DFG) for a Heisenberg fellowship, and RV acknowledges partial support by CICYT under contract AEN95-0882. 


\section{References}

[1] F.Y. Wu, Rev. Mod. Phys. 54 (1982) 235; 55 (1983) 315(E).

[2] J.P. Straley, J. Phys. A7 (1974) 2173.

[3] D. Kim and R.I. Joseph, J. Phys. A8 (1975) 891.

[4] S. Miyashita, D.D. Betts, and C.J. Elliott, J. Phys. A12 (1979) 1605.

[5] H.W.J. Blöte and R.H. Swendsen, Phys. Rev. Lett. 43 (1979) 799.

[6] H.J. Herrmann, Z. Phys. B35 (1979) 171.

[7] S.J. Knak-Jensen and O.G. Mouritsen, Phys. Rev. Lett. 43 (1979) 1736.

[8] I. Ono and K. Ito, J. Phys. C15 (1982) 4417.

[9] W.G. Wilson and C.A. Vause, Phys. Rev. B36 (1987) 587.

[10] R.V. Gavai, F. Karsch, and B. Petersson, Nucl. Phys. B322 (1989) 738.

[11] M. Fukugita, H. Mino, M. Okawa, and A. Ukawa, J. Stat. Phys. 59 (1990) 1397.

[12] N.A. Alves, B.A. Berg, and R. Villanova, Phys. Rev. B43 (1991) 5846.

[13] A. Yamagata, J. Phys. A26 (1993) 2091.

[14] M. Fukugita and M. Okawa, Phys. Rev. Lett. 63 (1989) 13.

[15] C.-K. Hu and K.-S. Mak, Phys. Rev. B40 (1989) 5007.

[16] A. Billoire, R. Lacaze, and A. Morel, Nucl. Phys. B340 (1990) 542.

[17] F. Karsch and A. Patkós, Nucl. Phys. B350 (1991) 563.

[18] O.F. De Alcantara Bonfim, J. Stat. Phys. 62 (1991) 105.

[19] J. Lee and J.M. Kosterlitz, Phys. Rev. B43 (1991) 1268.

[20] J.-D. Wang and C. DeTar, Phys. Rev. D47 (1993) 4091. 
[21] K. Vollmayr, J.D. Reger, M. Scheucher, and K. Binder, Z. Phys. B91 (1993) 113.

[22] M. Schmidt, Z. Phys. B95 (1994) 327.

[23] P. Provero and S. Vinti, Physica A211 (1994) 436.

[24] M.E. Fisher and A.N. Berker, Phys. Rev. B26 (1982) 2507; V. Privman and M.E. Fisher, J. Stat. Phys. 33 (1983) 385; K. Binder and D.P. Landau, Phys. Rev. B30 (1984) 1477; M.S.S. Challa, D.P. Landau, and K. Binder, Phys. Rev. B34 (1986) 1841; V. Privman and J. Rudnik, J. Stat. Phys. 60 (1990) 551; V. Privman, in Finite-Size Scaling and Numerical Simulations of Statistical Physics, edited by V. Privman (World Scientific, Singapore, 1990), p. 1.

[25] C. Borgs and R. Kotecky, J. Stat. Phys. 61 (1990) 79; Phys. Rev. Lett. 68 (1992) 1734; C. Borgs, R. Kotecky, and S. Miracle-Solé, J. Stat. Phys. 62 (1991) 529.

[26] H.J. Herrmann, W. Janke, and F. Karsch (eds.), Dynamics of First Order Phase Transitions (World Scientific, Singapore, 1992).

[27] W. Janke, in Computer Simulations in Condensed Matter Physics VII, eds. D.P. Landau, K.K. Mon, and H.-B. Schüttler (Springer, Berlin 1994), p. 29.

[28] C. Borgs and W. Janke, Phys. Rev. Lett. 68 (1992) 1738.

[29] W. Janke, Phys. Rev. B47 (1993) 14757.

[30] B.A. Berg and T. Neuhaus, Phys. Lett. B267 (1991) 249; Phys. Rev. Lett. 68 (1992) 9; W. Janke, B.A. Berg, and M. Katoot, Nucl. Phys. B382 (1992) 649.

[31] W. Janke and S. Kappler, Phys. Rev. Lett. 74 (1995) 212.

[32] A.M. Ferrenberg and R.H. Swendsen, Phys. Rev. Lett. 61 (1988) 2635; ibid. 63 (1989) 1658(E).

[33] R.G. Miller, Biometrika 61 (1974) 1; B. Efron, The Jackknife, the Bootstrap and other Resampling Plans (SIAM, Philadelphia, PA, 1982). 
[34] K. Binder, Phys. Rev. A25 (1982) 1699; Z. Phys. B43 (1981) 119.

[35] W. Janke and S. Kappler, to be published.

[36] J. Lee and J.M. Kosterlitz, Phys. Rev. B43 (1991) 3265.

[37] W. Janke, unpublished.

[38] T. Bhattacharya, R. Lacaze, and A. Morel, Saclay preprint SPhT96/001, hep-lat/9601012 (January 1996).

[39] T. Bhattacharya, R. Lacaze, and A. Morel, Europhys. Lett. 23 (1993) 547; Nucl. Phys. B (Proc. Suppl.) 34 (1994) 671.

[40] A. Billoire, R. Lacaze, and A. Morel, Nucl. Phys. B370 (1992) 773.

[41] W. Janke, Mainz preprint (May 1996).

[42] W. Janke and S. Kappler, Europhys. Lett. 31 (1995) 345; Nucl. Phys. B (Proc. Suppl.) 42 (1995) 770.

[43] M.S. Carroll, W. Janke, and S. Kappler, to be published.

[44] J. de Coninck, A. Messager, S. Miracle-Solé, and J. Ruiz, J. Stat. Phys. 52 (1988) 45; R.H. Schonmann, J. Stat. Phys. 52 (1988) 61. 


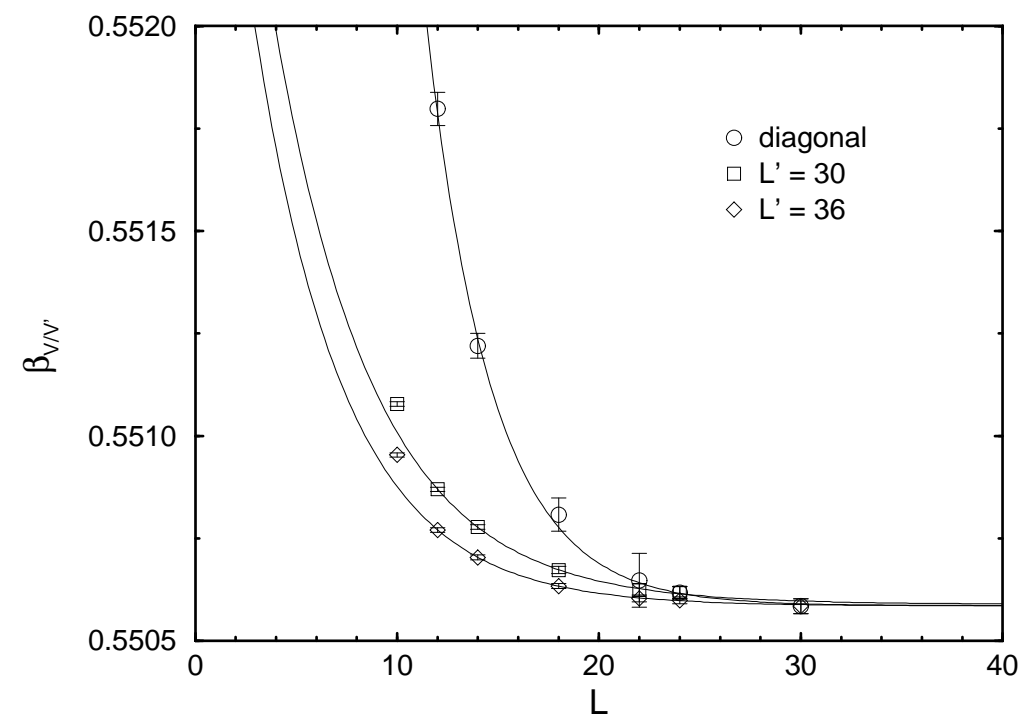

Fig. 1

Figure 1: Finite-size scaling of the pseudo-transition points $\beta_{V / V^{\prime}}$ of the number-of-phases criterion together with exponential fits of the form $\beta_{V / V^{\prime}}=$ $\beta_{t}+a \exp (-b L)$.

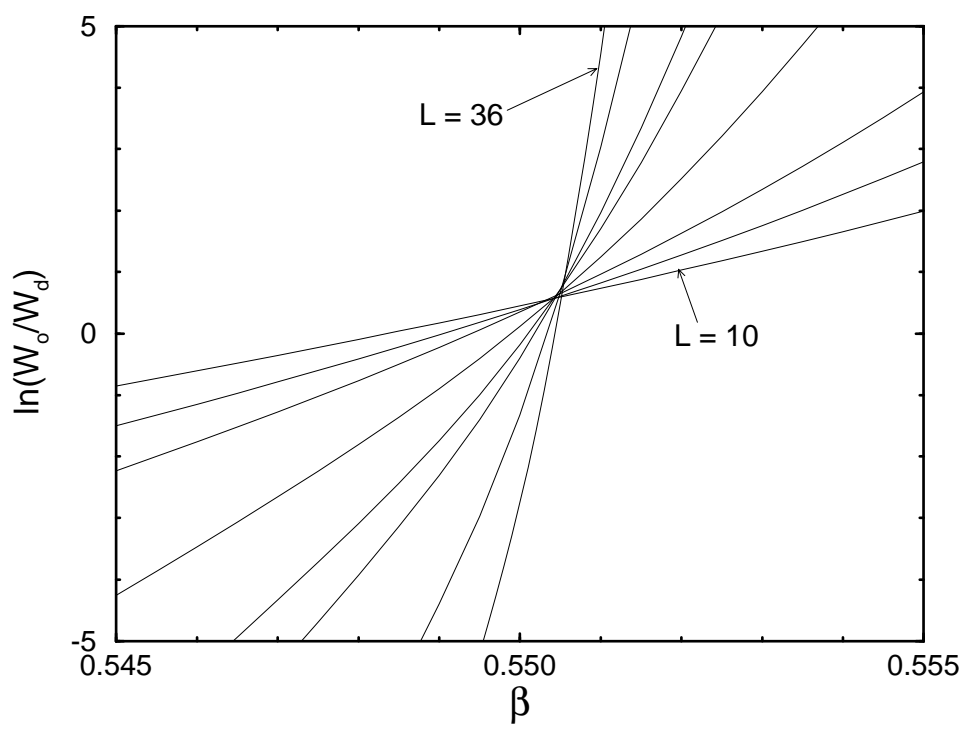

Fig. 2

Figure 2: The logarithm of the weight ratio as a function of the inverse temperature for lattices of size $L=10,12,14,18$, 22, 24, 30, and 36 . 


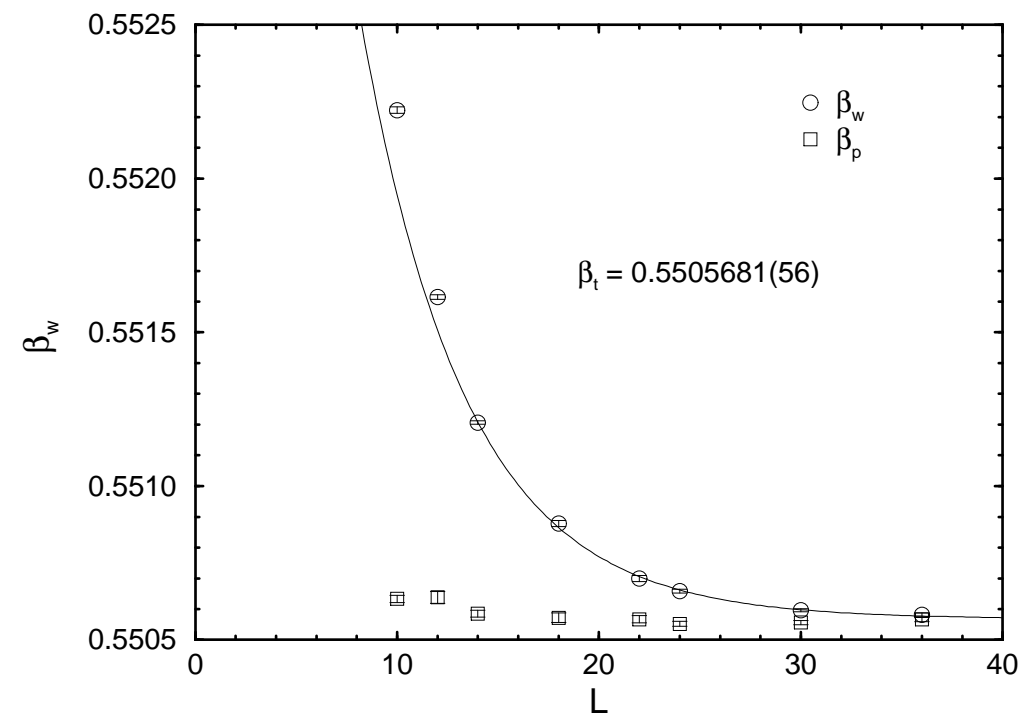

Fig. 3

Figure 3: Finite-size scaling of the pseudo-transition points $\beta_{w}$ of the ratioof-weights method together with an exponential fit of the form $\beta_{w}=\beta_{t}+$ $a \exp (-b L)$. Also shown are the points $\beta_{p}$ where the two peaks of the energy probability distribution are of equal height.

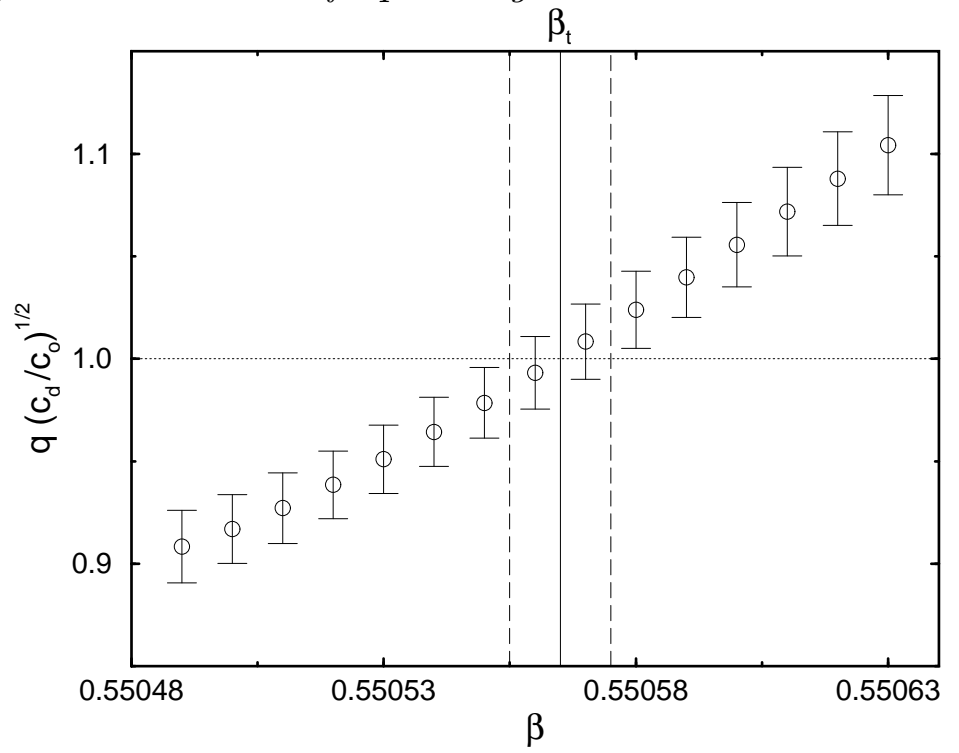

Fig. 4

Figure 4: Theoretical prediction (16) for the height ratio as a function of the inverse temperature. The vertical lines show the infinite-volume transition point together with the statistical error bounds. 


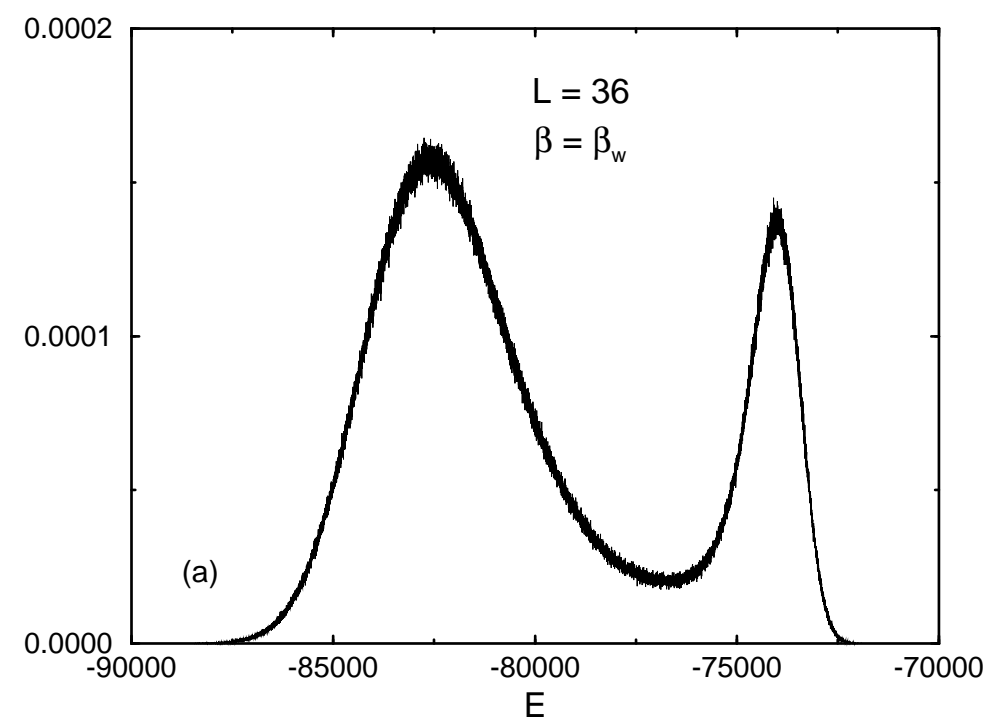

Fig. $5 a$

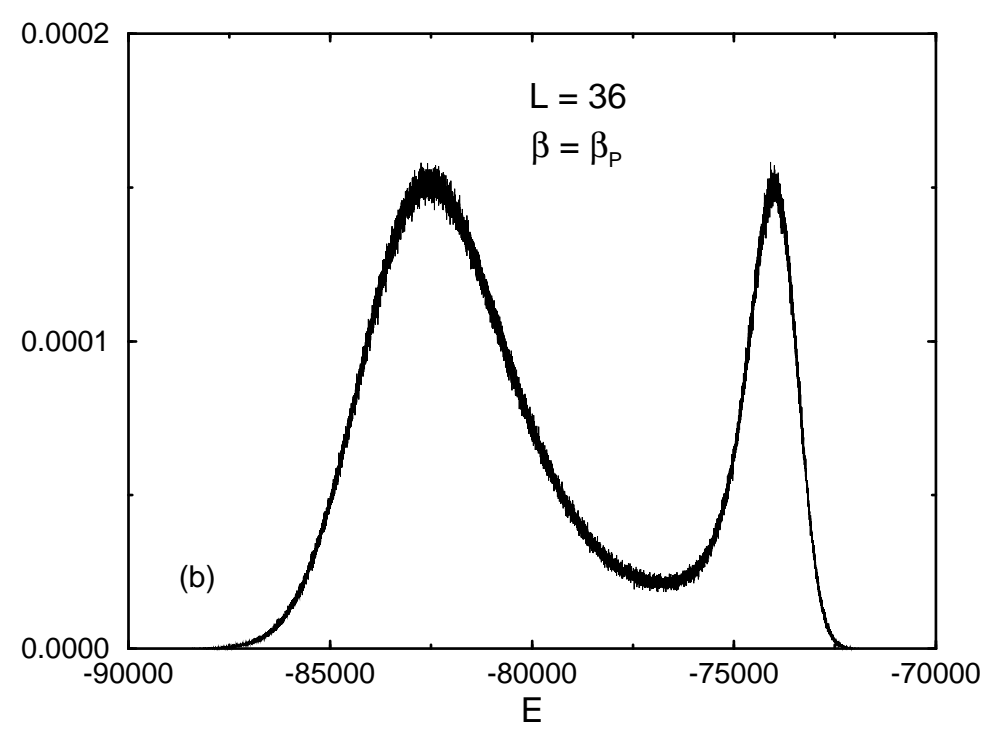

Fig. $5 \mathrm{~b}$

Figure 5: Histogram for $L=36$ reweighted to (a) $\beta_{w}(L=36)=0.5505812$ and (b) $\beta_{p}(L=36)=0.5505665$. 


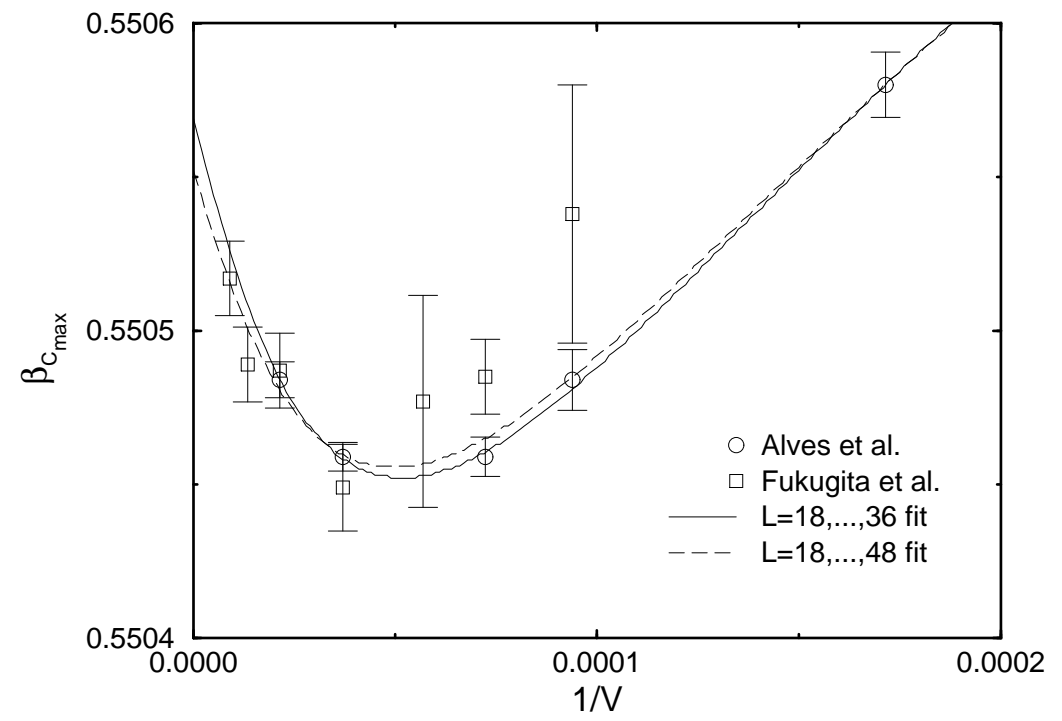

Fig. 6

Figure 6: Finite-size scaling of the specific-heat maxima locations together with fits of the form $\beta_{C_{\max }}=\beta_{t}+c / V+a \exp (-b L)$.

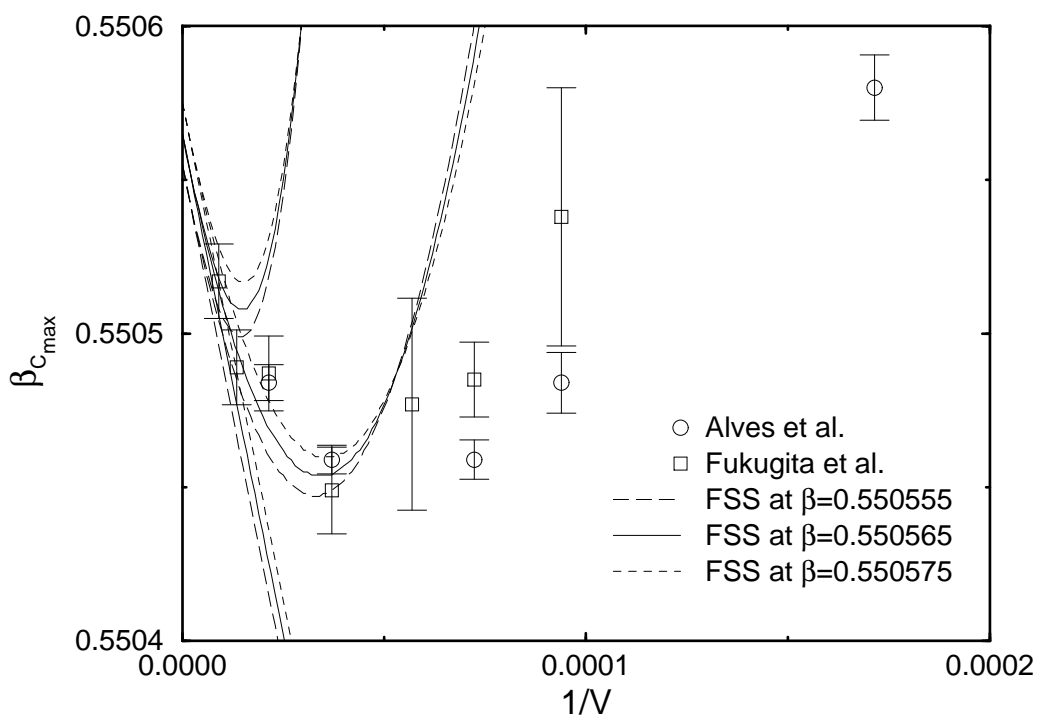

Fig. 7

Figure 7: Finite-size scaling of the specific-heat maxima locations together with the theoretically predicted FSS power-law expansion in $1 / V$ to first, second, and third order (see text). 


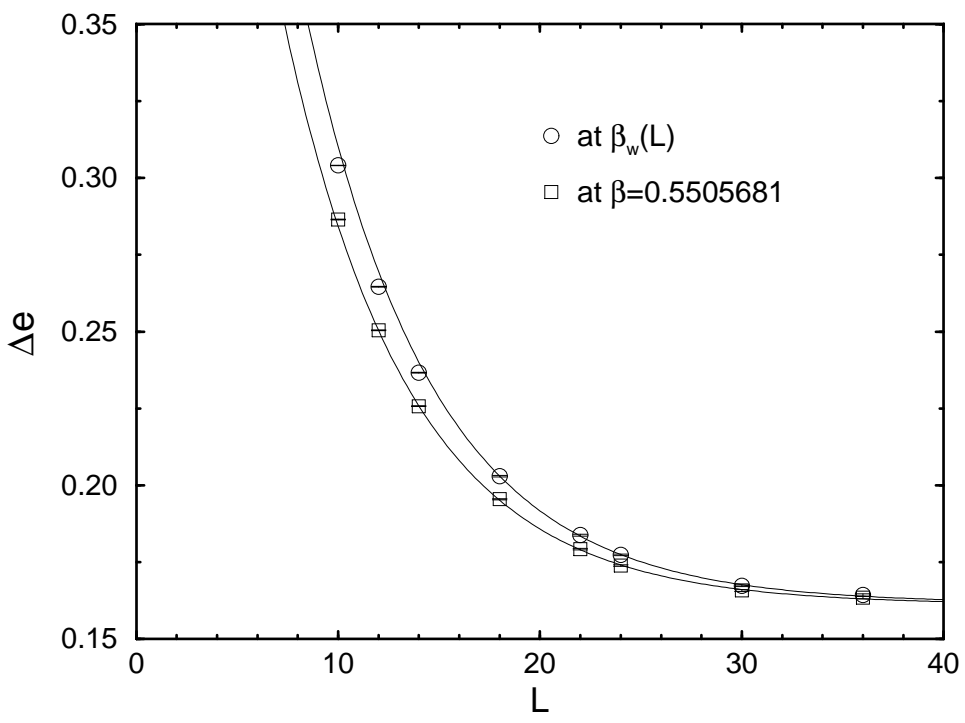

Fig. 8

Figure 8: Finite-size scaling of the latent heat derived from the ratio-ofweights method. The continuous lines are fits of the form $\Delta e(L)=\Delta e(\infty)+$ $a \exp (-b L)$. 